\title{
Trajectory Planning for the Walking Biped "Lucy"
}

\author{
Jimmy Vermeulen ${ }^{1}$, Dirk Lefeber, Björn Verrelst and Bram Vanderborght \\ ${ }^{1}$ Vrije Universiteit Brussel, Pleinlaan 2, Brussels, Belgium \\ Jimmy.Vermeulen@vub.ac.be \\ http://lucy.vub.ac.be/trajectory.html
}

Summary. A real-time joint trajectory generation strategy for the dynamic walking biped "Lucy" $[1,2]$ is proposed. This trajectory planner generates dynamically stable motion patterns by using a set of objective locomotion parameters as its input, and by tuning and exploiting the natural upper body dynamics. The latter can be determined and manipulated by using the angular momentum equation. Basically, trajectories for hip and swing foot motion are generated, which guarantee that the objective locomotion parameters attain certain prescribed values. Additionally, the hip trajectories are slightly modified such that the upper body motion is steered naturally, meaning that it requires practically no actuation. This has the advantage that the upper body actuation hardly influences the position of the ZMP. The effectiveness of the developed strategy is proven by simulation results.

\section{Introduction}

The upper body of a biped or a humanoid robot usually contains the on-board hardware of the control system, as well as the batteries in case of electric actuation, or a pressurized vessel or a compressor in case of pneumatic actuation. Therefore the upper body is generally the robot link with the largest mass and inertia. As a consequence, keeping this body upright requires significant action of the actuators, which might cause problems concerning the position of the Zero Moment Point [3]. In this paper, a planning strategy is developed that uses the angular momentum equation to estimate the natural dynamics of the upper body, or in other words, the motion of the upper body when it is unactuated. The trajectories for the leg links are then established such that this natural behaviour of the upper body approximates a given prescribed behaviour. When this desired behaviour, which will be modelled by a polynomial function, is used as a reference trajectory for the upper body actuator, then the work of this actuator is limited to overcoming the minor differences between the natural and the polymomial trajectory. The advantage of this strategy is that the resulting motion of the ZMP stays well within the boundaries of the stability region during the whole locomotion process. During the 
single support phase the ZMP remains in the near vicinity of the ankle joint of the supporting foot. During the double support phase the ZMP travels from the rear ankle to the front ankle joint.

A planar walking biped "Lucy" has been developed by the Multibody Mechanics Research Group at the Vrije Universiteit Brussel [1]. The trajectory planner described in this paper is tested by simulation on the model of Lucy. Since its effectiveness has been verified, this strategy will be implemented on the real robot in the near future. The simulated motion of Lucy contains single as well as double support phases, while also taking the impact phase into account. The strategy developed consists of a limited number of elementary computations, which makes it useful as a real-time trajectory planner for the biped Lucy.

\section{Double Support Phase}

In figure 1 the model of the planar biped Lucy is depicted during a double support phase. The R stands for Rear foot, whereas the F stands for Front foot. Since both feet are in contact with the ground, a closed kinematic chain

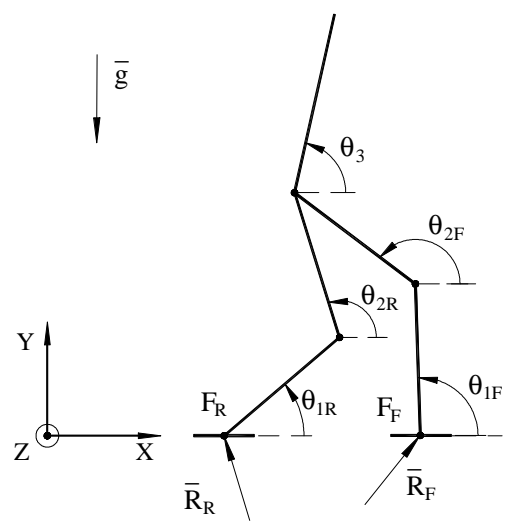

Fig. 1. Lucy during DSP

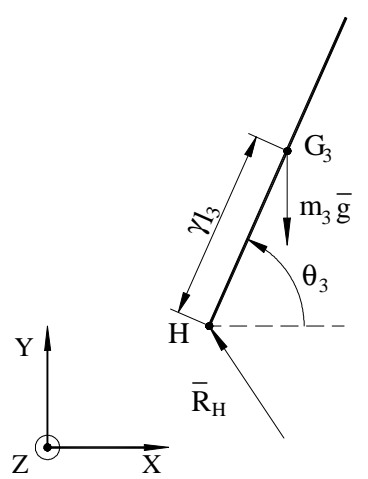

Fig. 2. FBD of upper body

is formed by the two legs and the ground. It is desired that both feet stay in contact with the ground and that the feet do not slip during a double support phase. The horizontal and vertical distances between the two ankle joints are respectively called step length and step height and are given by:

$$
\begin{aligned}
X_{F_{F}}-X_{F_{R}}=\lambda & \text { (step length) } \\
Y_{F_{F}}-Y_{F_{R}}=\delta & \text { (step heigth) }
\end{aligned}
$$

Due to the two holonomic constraints, the robot's number of DOF is equal to three. 


\subsection{Hip Motion during DSP}

Suppose that the following Lagrange coordinates are chosen to describe the motion:

$$
q_{D}=\left(X_{H}, Y_{H}, \theta_{3}\right)
$$

where $X_{H}$ and $Y_{H}$ respectively represent the horizontal and vertical position of the hip joint. In order to obtain smooth joint trajectories for the leg links, fifth order polynomial functions are established to be tracked during the double support phase. Suppose initially that only the two knee actuators are used. The polynomial functions have to connect the following initial and final boundary values for the hip motion:

$$
\begin{array}{rrrrrr}
X_{H}\left(t_{+}\right), \quad \dot{X}_{H}\left(t_{+}\right), \quad \ddot{X}_{H}\left(t_{+}\right) & \rightarrow & X_{H}\left(t_{D}\right), \quad \dot{X}_{H}\left(t_{D}\right), \quad \ddot{X}_{H}\left(t_{D}\right) \\
Y_{H}\left(t_{+}\right), \quad \dot{Y}_{H}\left(t_{+}\right), \quad \ddot{Y}_{H}\left(t_{+}\right) & \rightarrow & Y_{H}\left(t_{D}\right), \quad \dot{Y}_{H}\left(t_{D}\right), \quad \ddot{Y}_{H}\left(t_{D}\right)
\end{array}
$$

where $t_{+}$indicates the time instance immediately after the impact phase, and $t_{D}$ represents the end of the double support phase. The duration of the double support phase is then equal to $T_{D}=t_{D}-t_{+}$. Note that if the robot starts the double support phase from rest, that $t_{+}=0$ can be chosen, combined with $\dot{X}_{H}(0)=0, \ddot{X}_{H}(0)=0, \dot{Y}_{H}(0)=0$ and $\ddot{Y}_{H}(0)=0$.

\subsection{Upper Body Motion during DSP}

In order to derive the natural motion of the upper body during the double support phase, it is assumed that no actuator torque is acting on it. In that case, the upper body behaves as an inverted pendulum with a moving supporting point, being the hip point $\mathrm{H}$. Considering the free body diagram of the upper body in figure 2, and applying the angular momentum theorem with respect to the hip point $\mathrm{H}$, yields:

$$
\dot{\bar{\mu}}_{H}=\overline{H G}_{3} \times m_{3} \bar{g}+m_{3}\left(\bar{v}_{G_{3}} \times \bar{v}_{H}\right)
$$

It can be shown that under the assumption of small rotations of the pendulum, equation (4) results in the following differential equation [4]:

$$
\ddot{\theta}_{3}=C\left[\ddot{X}_{H}-\left(\ddot{Y}_{H}+g\right)\left(\frac{\pi}{2}-\theta_{3}\right)\right] \quad \text { with } \quad C=\frac{m_{3} \gamma l_{3}}{I_{3}+\gamma^{2} l_{3}^{2} m_{3}}
$$

and $g$ representing gravity acceleration and $I_{3}$ being the moment of inertia of the upper body with respect to its COG.

When $X_{H}(t)$ and $Y_{H}(t)$ are given functions of time, and when initial conditions $\dot{\theta}_{3}\left(t_{+}\right)$and $\theta_{3}\left(t_{+}\right)$are specified, this equation can be numerically integrated to obtain the natural upper body motion during the double support phase. However, the goal here is not to determine a natural motion exactly, but to develop a trajectory for the upper body angle which corresponds to a 
motion close to a natural motion. A rough approximation of the natural upper body motion can be found by considering only the horizontal hip motion (by assuming that $\left.\theta_{3} \approx \frac{\pi}{2}\right)$ :

$$
\ddot{\theta}_{3}^{n a t}=C \ddot{X}_{H}
$$

By twice integrating (6) over time, one obtains:

$$
\begin{aligned}
\theta_{3}^{\text {nat }}(t)= & \theta_{3}\left(t_{+}\right)+\left(t-t_{+}\right) \dot{\theta}_{3}\left(t_{+}\right) \\
& +C\left[X_{H}(t)-X_{H}\left(t_{+}\right)-\left(t-t_{+}\right) \dot{X}_{H}\left(t_{+}\right)\right]
\end{aligned}
$$

Suppose now that the duration of the double support phase is given the following value:

$$
T_{D}=\frac{X_{H}\left(t_{D}\right)-X_{H}\left(t_{+}\right)}{\dot{X}_{H}\left(t_{+}\right)}
$$

then roughly speaking the upper body angle at the end of the double support phase is equal to:

$$
\theta_{3}^{\text {nat }}\left(t_{D}\right)=\theta_{3}\left(t_{+}\right)+T_{D} \dot{\theta}_{3}\left(t_{+}\right)
$$

A fifth order polynomial function will be established for the upper body angle, connecting the following initial and final boundary values:

$$
\theta_{3}\left(t_{+}\right), \quad \dot{\theta}_{3}\left(t_{+}\right), \quad \ddot{\theta}_{3}\left(t_{+}\right) \quad \rightarrow \quad \theta_{3}\left(t_{D}\right), \quad \dot{\theta}_{3}\left(t_{D}\right), \quad \ddot{\theta}_{3}\left(t_{D}\right)
$$

with

$$
\begin{aligned}
& \theta_{3}\left(t_{D}\right)=\theta_{3}^{\text {nat }}\left(t_{D}\right) \\
& \ddot{\theta}_{3}\left(t_{D}\right)=C\left[\ddot{X}_{H}\left(t_{D}\right)-\left(\ddot{Y}_{H}\left(t_{D}\right)+g\right)\left(\frac{\pi}{2}-\theta_{3}^{n a t}\left(t_{D}\right)\right)\right]
\end{aligned}
$$

A suitable value for the angular velocity $\dot{\theta}_{3}\left(t_{D}\right)$ will be calculated in section 3.2 , where calculations for the single support phase are performed.

\section{Single Support Phase}

In figure 3 the biped Lucy is depicted during a single support phase. The S stands for supporting leg, whereas the A stands for swing (air) leg. Since it is assumed that the supporting foot stays in contact with the ground and does not slip during a single support phase, the number of DOF is equal to five.

\subsection{Hip and Swing Foot Motion during SSP}

Suppose that the following Lagrange coordinates are chosen to describe the motion:

$$
q_{S}=\left(X_{H}, Y_{H}, X_{F_{A}}, Y_{F_{A}}, \theta_{3}\right)
$$




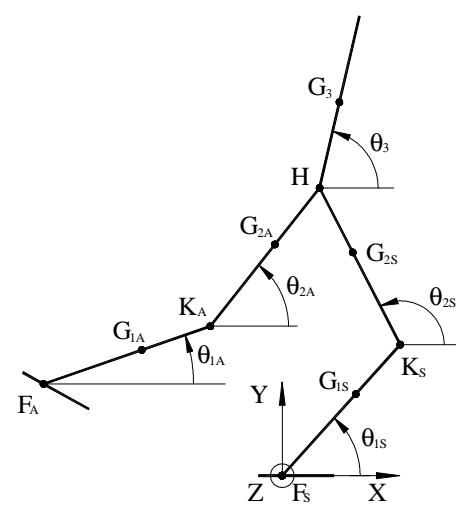

Fig. 3. Lucy during single support phase

Assuming initially that no external ankle torque is exerted, so that only the knee and hip actuators are used, the robot is an underactuated mechanism. Two fifth order polynomial functions for the leg links of the supporting leg are established, which connect the following initial and final boundary values for the hip motion:

$$
\begin{array}{rrrrrr}
X_{H}\left(t_{D}\right), \quad \dot{X}_{H}\left(t_{D}\right), \quad \ddot{X}_{H}\left(t_{D}\right) & \rightarrow & X_{H}\left(t_{S}\right), \quad \dot{X}_{H}\left(t_{S}\right), \quad \ddot{X}_{H}\left(t_{S}\right) \\
Y_{H}\left(t_{D}\right), \quad \dot{Y}_{H}\left(t_{D}\right), \quad \ddot{Y}_{H}\left(t_{D}\right) & \rightarrow & Y_{H}\left(t_{S}\right), \quad \dot{Y}_{H}\left(t_{S}\right), \quad \ddot{Y}_{H}\left(t_{S}\right)
\end{array}
$$

where $t_{S}$ represents the end of the single support phase. The duration of the single support phase is then equal to $T_{S}=t_{S}-t_{D}$. This single support phase duration is defined as:

$$
T_{S}=\frac{X_{H}\left(t_{S}\right)-X_{H}\left(t_{D}\right)}{\nu}
$$

where $\nu$ is an objective locomotion parameter defining the mean horizontal hip velocity during a single support phase.

Two sixth order polynomial functions for the leg links of the swing leg are established, which connect the following initial, intermediate and final boundary values for the swing foot motion:

$$
\begin{aligned}
X_{F_{A}}\left(t_{D}\right), \dot{X}_{F_{A}}\left(t_{D}\right), \ddot{X}_{F_{A}}\left(t_{D}\right) & \rightarrow \quad X_{F_{A}}\left(t_{i}\right) \rightarrow X_{F_{A}}\left(t_{S}\right), \dot{X}_{F_{A}}\left(t_{S}\right), \ddot{X}_{F_{A}}\left(t_{S}\right) \\
Y_{F_{A}}\left(t_{D}\right), \dot{Y}_{F_{A}}\left(t_{D}\right), \ddot{Y}_{F_{A}}\left(t_{D}\right) & \rightarrow \quad Y_{F_{A}}\left(t_{i}\right) \rightarrow Y_{F_{A}}\left(t_{S}\right), \dot{Y}_{F_{A}}\left(t_{S}\right), \ddot{Y}_{F_{A}}\left(t_{S}\right)
\end{aligned}
$$

The intermediate condition at $t=t_{i}$ is used to lift the foot whenever an obstacle has to be cleared during the swing phase. Note also that in all cases

$$
\dot{X}_{F_{A}}\left(t_{D}\right)=\ddot{X}_{F_{A}}\left(t_{D}\right)=0=\dot{Y}_{F_{A}}\left(t_{D}\right)=\ddot{Y}_{F_{A}}\left(t_{D}\right)
$$

since during the double support phase the feet remain fixed to the ground. 


\subsection{Upper Body Motion during SSP}

In order to obtain the natural upper body motion, it is initially assumed that the ankle actuator is not used. In that case one can write the angular momentum equation with respect to the ankle joint of the supporting foot as follows:

$$
\dot{\mu}_{F_{S}}=-M g\left(X_{G}-X_{F_{S}}\right)
$$

where $X_{G}$ is the horizontal position of the global COG.

The kinematical expression of the angular momentum can be written as:

$$
\mu_{F_{S}}=A_{3} \dot{\theta}_{3}+h
$$

with the function $h$ being independent of the angular velocity of the upper body $\dot{\theta}_{3}$. The complete expressions for the functions $h$ and $A_{3}$ can be found in [4] (see http://lucy.vub.ac.be/trajectory.html).

\section{Upper body angle}

Integrating (14) from $u=t_{D}$ to $u=t$, gives:

$$
\mu_{F_{S}}(t)-\mu_{F_{S}}\left(t_{D}\right)=-M g \int_{t_{D}}^{t} X_{G} d u
$$

A second integration from $t=t_{D}$ to $t=t_{S}$ yields:

$$
\int_{t_{D}}^{t_{S}} \mu_{F_{S}}(t) d t-\mu_{F_{S}}\left(t_{D}\right) T_{S}=-M g \int_{t_{D}}^{t_{S}}\left(T_{S}-t\right) X_{G} d t
$$

Now introducing (15) into the lhs of (17) and solving for $\dot{\theta}_{3}\left(t_{D}\right)$ gives:

$$
\dot{\theta}_{3}\left(t_{D}\right)=F+\frac{1}{T_{S} A_{3}\left(t_{D}\right)} \int_{t_{D}}^{t_{S}} A_{3} \dot{\theta}_{3} d t
$$

with

$$
F=\frac{1}{A_{3}\left(t_{D}\right)}\left[-M g \int_{t_{D}}^{t_{S}}\left(T_{S}-t\right) X_{G} d t+h\left(t_{D}\right) T_{S}-\int_{t_{D}}^{t_{S}} h d t\right]
$$

It can be shown [4] that when assuming small rotations of the upper body in the neighborhood of $\frac{\pi}{2}$, as well as small vertical motions of the hip joint, the function $A_{3}$ can be approximated as a constant: 


$$
A_{3}(t) \approx I_{3}+m_{3} \gamma^{2} l_{3}^{2}+m_{3} \gamma l_{3} Y_{H}(t) \approx A_{3}\left(t_{D}\right) \quad\left(t_{D} \leq t \leq t_{S}\right)
$$

Expression (18) then becomes:

$$
\dot{\theta}_{3}\left(t_{D}\right)=F+\frac{\theta_{3}\left(t_{S}\right)-\theta_{3}\left(t_{D}\right)}{T_{S}}=F+\frac{\Delta \theta_{3}^{S}}{T_{S}}
$$

Now recalling expression (9), which calculates the upper body rotation during the double support phase:

$$
\Delta \theta_{3}^{D}=T_{D} \dot{\theta}_{3}\left(t_{+}\right)
$$

and demanding that $\Delta \theta_{3}^{D}+\Delta \theta_{3}^{S}=0$ allows one to determine a necessary value for $\dot{\theta}_{3}\left(t_{D}\right)$ :

$$
\dot{\theta}_{3}\left(t_{D}\right)=F-\frac{T_{D}}{T_{S}} \dot{\theta}_{3}\left(t_{+}\right)
$$

If this specific value for the angular velocity of the upper body is used for the construction of the polynomial function for the upper body during the preceding double support phase (see section 2.2), then the upper body rotation will be compensated during the next single support phase, without the use of an ankle actuator.

\section{Upper body angular velocity}

Evaluating (16) at $t=t_{S}$ and introducing the kinematical expression (15) yields:

$$
\dot{\theta}_{3}\left(t_{S}\right)=\dot{\theta}_{3}\left(t_{D}\right)+\frac{1}{A_{3}\left(t_{D}\right)}\left[h\left(t_{D}\right)-h\left(t_{S}\right)-M g \int_{t_{D}}^{t_{S}} X_{G} d t\right]
$$

By varying the integral on the rhs of equation (24), different values for $\dot{\theta}_{3}\left(t_{S}\right)$ can be obtained. In practice a specific angular velocity $\dot{\theta}_{3}\left(t_{S}\right)$ can e.g. be attained by iteratively shifting the horizontal position of the hip point $X_{H}\left(t_{D}\right)$ at the end of the double support phase.

\section{Upper body angular acceleration}

Evaluating (14) at $t=t_{D}$ and introducing the kinematical expression (15), gives:

$$
A_{3}\left(t_{D}\right) \ddot{\theta}_{3}\left(t_{D}\right)+\dot{A}_{3}\left(t_{D}\right) \dot{\theta}_{3}\left(t_{D}\right)+\dot{h}\left(t_{D}\right)=-M g\left[X_{G}\left(t_{D}\right)-X_{F_{S}}\left(t_{D}\right)\right]
$$

Note that this equation corresponds to a zero ankle torque, or in other words to a ZMP located exactly at the ankle joint. Since $\ddot{\theta}_{3}\left(t_{D}\right)$ is imposed by the polynomial function during the double support phase, introducing expression 
(11) in (25) yields a condition which has to be satisfied at the beginning of the single support phase. Satisfying this equation results in a transition from double to single support phase with a ZMP coinciding with the ankle joint. In practice this can e.g. be achieved by tuning the hip accelerations $\ddot{X}_{H}\left(t_{D}\right)$ and $\ddot{Y}_{H}\left(t_{D}\right)$. For example, if one chooses a specific value for $\ddot{X}_{H}\left(t_{D}\right)(<0)$, then $(25)$ combined with (11) can be solved for $\ddot{Y}_{H}\left(t_{D}\right)$.

An analogous reasoning can be done at the end of the single support phase $t=t_{S}$, yielding a condition on $\ddot{X}_{H}\left(t_{S}\right)$ and $\ddot{Y}_{H}\left(t_{S}\right)$. This condition has to be satisfied in order to have the ZMP located at the ankle joint of the supporting foot before the impact occurs.

\section{Tracking Function}

In the preceding paragraphs, the natural upper body motion was manipulated such that the upper body is steered without requiring an ankle actuator, which corresponds to a ZMP located at the ankle joint during the single support phase. In order to compensate for modelling errors and possible external disturbances, a fifth order polynomial function will be established. This polynomial function is constructed with the boundary values from a natural motion, meaning that it is a reasonable approximation of the natural motion. It connects the following boundary values:

$$
\theta_{3}\left(t_{D}\right), \quad \dot{\theta}_{3}\left(t_{D}\right), \quad \ddot{\theta}_{3}\left(t_{D}\right) \quad \rightarrow \quad \theta_{3}\left(t_{S}\right), \quad \dot{\theta}_{3}\left(t_{S}\right), \quad \ddot{\theta}_{3}\left(t_{S}\right)
$$

Consequently, the torque exerted by the ankle actuator is low, meaning that the ZMP remains in the near vicinity of the ankle joint, which is the most dynamically stable position. Note also that due to the developed strategy during the double support phase, the ZMP automatically transfers from the rear ankle to the front ankle, without requiring external torques. Indeed, polynomial trajectories are constructed, connecting two successive single support phases, each with the ZMP located exactly at the ankle joint of its supporting foot.

\section{Simulation}

To test the effectiveness of the trajectory planner, a number of different simulations were performed [4]. The results of one steady walking pattern where Lucy walks on a stairway are briefly reported here. The following objective parameters form the input for the trajectory planner:

- mean horizontal hip velocity $\nu=0.5 \frac{\mathrm{m}}{\mathrm{s}}$

- step length $\lambda=0.3 \mathrm{~m}$

- step height $\delta=0.1 \mathrm{~m}$

The total step duration is approximately $0.58 s$, with a single support duration $T_{S}=0.46 \mathrm{~s}$, and a double support duration $T_{D}=0.12 \mathrm{~s}$. 
Figures 4 and 5 show respectively the horizontal and vertical hip position during 1 full step, whereas figures 6 and 7 show respectively the horizontal and vertical swing foot trajectory during a single support phase. For this simulation, expression (23) yields $\dot{\theta}_{3}\left(t_{D}\right)=-0.19 \frac{\mathrm{rad}}{\mathrm{s}}$, which results in $\Delta \theta_{3}^{S}=$ $-\Delta \theta_{3}^{D}=0.022 \mathrm{rad}$. In expression $(24) \dot{\theta}_{3}\left(t_{S}\right)=\dot{\theta}_{3}\left(t_{D}\right)$ was chosen, which results in $X_{H}\left(t_{D}\right)-X_{F_{S}}\left(t_{D}\right)=-0.133 \mathrm{~m}$ at the beginning of the single support phase.

In expression $(25)$, the value $\ddot{Y}_{H}\left(t_{D}\right)=0$ was chosen, yielding $\ddot{X}_{H}\left(t_{D}\right)=$ $-2.4 \frac{\mathrm{m}}{\mathrm{s}^{2}}$. These accelerations guarantee that the ZMP is located exactly at the ankle joint of the supporting foot. An analogue calculation is performed at the end of the single support phase. Choosing $\ddot{Y}_{H}\left(t_{S}\right)=-3.5 \frac{\mathrm{m}}{\mathrm{s}^{2}}$, leads to $\ddot{X}_{H}\left(t_{S}\right)=1.4 \frac{m}{s^{2}}$. The polynomial trajectory for the upper body angle which

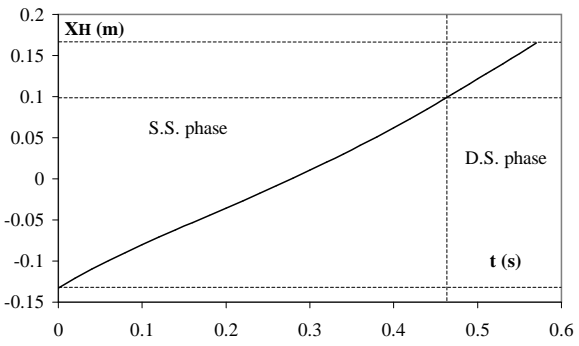

Fig. 4. Horizontal hip trajectory

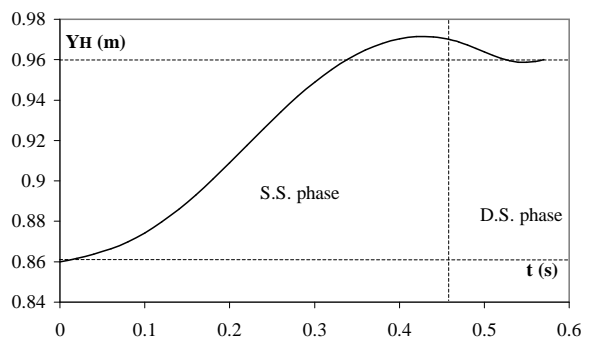

Fig. 5. Vertical hip trajectory

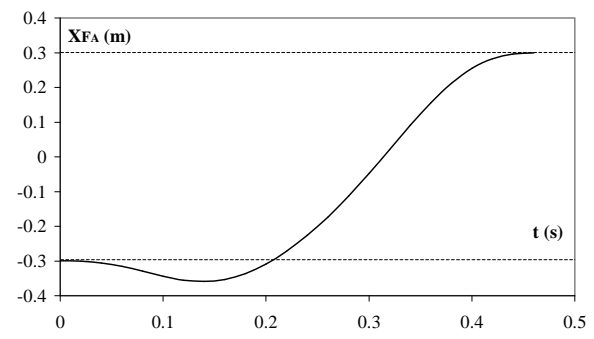

Fig. 6. Horizontal swing foot trajectory

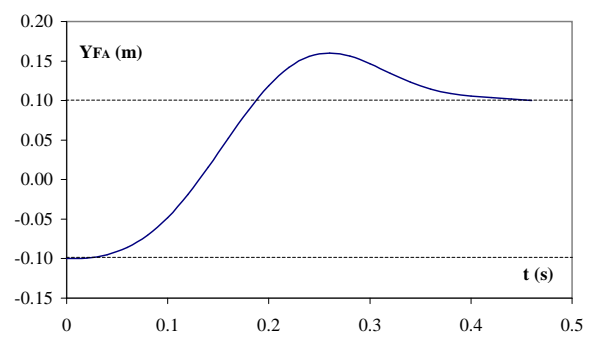

Fig. 7. Vertical swing foot trajectory

approximates a natural trajectory, is shown in figure 8. It can be verified that the overall upper body oscillation is very small, although practically no actuation is required to achieve this. The corresponding trajectory of the ZMP is given in figure 9. Indeed during the single support phase the ZMP is located in the near vicinity of the ankle joint. During the double support phase the ZMP travels from the rear ankle to the front ankle joint. This motion of the ZMP is caused by the trajectory planner, which generates trajectories for the 


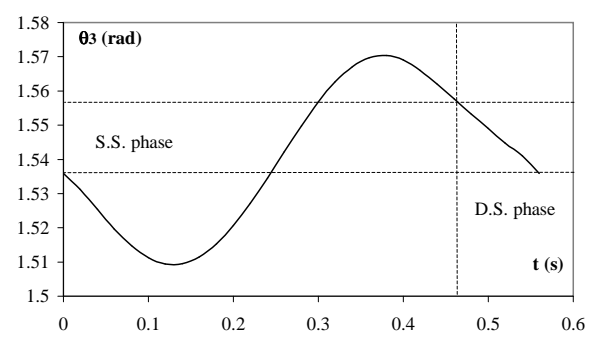

Fig. 8. Upper body angle

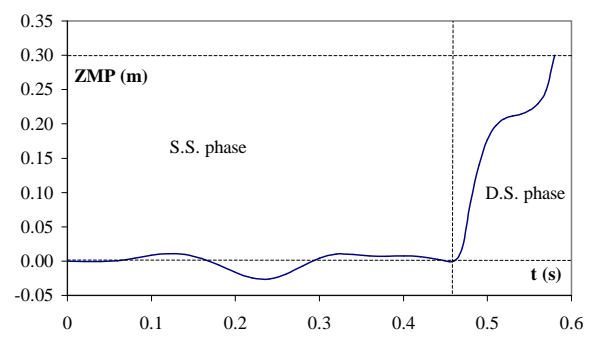

Fig. 9. Horizontal position of ZMP

leg links by a priori taking the ZMP position into account. This is in fact an open-loop ZMP control strategy.

\section{Conclusions}

A trajectory planner for the planar walking biped Lucy has been developed. A set of objective locomotion parameters form the input for this planner, while the output are polynomial tracking functions for each of the robot links. The strategy tunes the natural upper body dynamics by manipulating the angular momentum equation, so that practically no actuation is required to keep the upper body upright. As a result, external ankle torques are extremely low, which means that the upper body actuation does not cause the ZMP to move out of the stability region. The leg link angle trajectories are established in such a way that the ZMP stays in the near vicinity of the ankle joint during the single support phase, and travels from the rear to the front ankle joint during the double support phase. Since the ZMP position is taken into account when establishing the tracking functions, the trajectory planner is in fact an open loop ZMP controller. The method is very effective, as can be seen by the simulation results. Since only a limited number of computations are performed, the strategy can be used in real time.

\section{References}

1. B. Verrelst, R. Van Ham, F. Daerden and D. Lefeber (2002) Design of a Biped Actuated by Pleated Artificial Muscles. In: Proceedings of the 5th International Conference on Climbing and Walking Robots (CLAWAR 2002), pp. 211-218

2. http://lucy.vub.ac.be

3. M. Vukobratovic and B. Borovac (2004) Zero-Moment Point - Thirty Five Years of its Life. International Journal of Humanoid Robotics (IJHR) 1(1):157-173

4. J. Vermeulen (2004) Trajectory Generation for Planar Hopping and Walking Robots: An Objective Parameter and Angular Momentum approach. PHD Thesis, Vrije Universiteit Brussel, Belgium 